\title{
SHORT REPORT \\ The pyogenic potential of the different Streptococcus anginosus group bacterial species: retrospective cohort study
}

\author{
O. $\mathrm{KOBO}^{1 *}$, S. NIKOLA ${ }^{2}$, Y. GEFFEN ${ }^{3}$ AND M. PAUL ${ }^{2,4}$ \\ ${ }^{1}$ Department of Internal Medicine C, Rambam Health Care Campus, Haifa, Israel \\ ${ }^{2}$ The Ruth and Bruce Rappaport Faculty of Medicine, Technion, Israel Institute of Technology, Haifa, Israel \\ ${ }^{3}$ The Microbiology Laboratory, Rambam Health Care Campus, Haifa, Israel \\ ${ }^{4}$ Infectious Diseases Institute, Rambam Health Care Campus, Haifa, Israel
}

Received 10 April 2017; Final revision 16 July 2017; Accepted 18 July 2017;

first published online 14 August 2017

\section{SUMMARY}

Streptococcus anginosus Group (SAG) bacteria are common causes of pyogenic infections (PIs). We examined the association between SAG species and the presence of a PI through a retrospective, observational, cohort study, between the years 2009 and 2015. All adults with clinically significant SAG infections in one hospital in Israel were assessed for association between SAG species and the presence of a PI defined as an abscess, empyema, or deep/organ space surgical site infection. Risk factors for PI were assessed using multivariate backward stepwise logistic regression analysis. We identified 263 patients with significant SAG infections, $182(69 \%)$ of which were caused by $S$. anginosus, $45(17 \cdot 1 \%)$ by Streptococcus constellatus and $36(13 \cdot 7 \%)$ by Streptococcus intermedius. The mean age of the patients was $56 \cdot 8 \pm 19 \cdot 1$ years. PIs were identified among $160(60 \%)$ of the patients and were mostly non-bacteraemic $(147 / 160,91 \cdot 8 \%)$, while most non-PI patients had bacteraemia (70/103, 68\%). S. anginosus and $S$. constellatus were associated with a significantly lower incidence of PI than $S$. intermedius, OR $0 \cdot 18(95 \%$ CI $0 \cdot 06-0 \cdot 53)$ and $0 \cdot 14(0 \cdot 04-0 \cdot 48)$, respectively. Patients with PI were younger and, in general, had less co-morbidities. S. intermedius was associated with pyogenic non-bacteraemic infections, while $S$. anginosus and $S$. constellatus were associated with bacteraemia with no abscess or empyema formation. These data may indicate differences in virulence mechanisms of these SAG bacteria.

Key words: Pyogenic infections, Streptococcus anginosus Group.

Streptococcus anginosus Group (SAG) infections are the most common streptococcal species causing pyogenic infections (PIs) and have variable clinical manifestations [1, 2]. Few data are available on the differences in clinical virulence of the different SAG species (S. anginosus, Streptococcus intermedius and

\footnotetext{
* Author for correspondence: O. Kobo, Department of Internal Medicine C, Rambam Health Care Campus, HaAliya HaShniya St 8, POB 9602, Haifa 31096, Israel.

(Email: oferkobo@yahoo.com)
}

Streptococcus constellatus) and their differential ability to cause a PI. In one retrospective cohort, 35\% of 245 SAG infections presented as a PI infection, with no reported differences between the species [3]. In another retrospective study of 118 patients with SAG infection, the proportion of patients with abscesses was $51 \%$ and $S$. anginosus was associated less frequently with abscess formation than the other SAG species [4]. S. intermedius has been linked to a worse prognosis, longer hospital stay [5] and disseminated PI [6]. However, these studies did not adjust for 
underlying clinical characteristics of patients infected with different SAG species.

Our objective was to examine retrospectively the association between SAG species and the presence of a PI in a relatively large cohort of patients over a 7-year period.

A retrospective, observational, cohort study was performed at Rambam Health Care Campus (RHCC) between the years 2009 and 2015. RHCC is a primary and tertiary care university-affiliated hospital of 960 beds, in northern Israel. The study was approved by the hospital's ethics committee with a waiver of informed consent given the observational nature of the study.

We included all consecutive adults ( $>18$ years) with clinically significant SAG infection. Patients were identified by the microbiology laboratory reports of all SAG isolates from all sites. From these, all patients fulfilling Systemic Inflammatory Response Syndrome criteria [7] accompanied by symptoms or signs of infection at the site of isolation or blood cultures positive for SAG were included. The dependent variable was the presence of a PI, defined as a deep tissue abscess, empyema, joint or bone infection or a surgical site infection (SSI) involving the operated organ or bone (organ/space SSI [8]) from which SAG was isolated (thereafter termed 'PI'). Other outcomes included disseminated infection, defined as a PI involving two or more organs, length of hospital stay, all-cause 30-day and in-hospital mortality. The exposure variable was the species of SAG; S. anginosus, $S$. intermedius or $S$. constellatus. We collected a large dataset of potential predictors for PIs including patients' demographics, background conditions, place of infection acquisition, infection presentation and presence of polymicrobial infection.

Data were collected from patients' medical records. RHCC operates a full electronic patient file that includes all clinical, laboratory and radiological data in the study years. Mortality data are updated by the national registry of the Ministry of Health.

SAG were identified to the species level using the Vitek 2 system (bioMerieux, Marcy l'Etoile, France).

We targeted a sample of at least 140 patients, assuming a $50 \%$ rate of PIs to include five independent variables in a multivariate analysis in addition to the exposure variable and compared between patients with and without pyogenic SAG infection. Categorical variables were compared using the $\chi^{2}$ test or the Fisher exact test, and continuous variables were compared using the $t$ test or the Mann-Whitney $U$-test, as appropriate. Variables found significant on univariate analysis $(P<0.05)$ were examined for statistical and clinical correlations and non-correlated variables were entered into a multivariate backward stepwise logistic regression analysis, in which the dependent variable was pyogenic infection. SAG species was forced into the analysis multiple imputations of blood urea nitrogen (BUN) were used for 19 patients to allow its inclusion in the regression model. Variables not contributing to the model's predictive ability were excluded from the final model. The goodness of fit and predictive ability of the model were examined using the Hosmer-Lemeshow and the receiver operating characteristics (ROC) curve, respectively. Analyses were performed using SPSS 20.

Over the study period, SAG bacteria were isolated from 301 adult patients, of which 263 had clinically significant infections, caused by $S$. anginosus in 182 patients $(69 \cdot 2 \%), S$. constellatus in $45(17 \cdot 1 \%)$ and $S$. intermedius in $36(13 \cdot 7 \%)$. Male predominance was noted $(168 / 263,63.9 \%)$. The mean age of the patients was $56 \cdot 8 \pm 19 \cdot 1$ years. All-cause 30-day and in-hospital mortality rates were $45 / 263(17 \cdot 1 \%)$ and $39 / 263$ (14.8\%), respectively.

PIs were identified among 160/263 (60\%) patients, and among these, $13(8 \cdot 1 \%)$ had bacteraemia and eight $(5 \%)$ had disseminated infection. PIs included intra-abdominal abscesses or peritonitis (69 patients, $43 \cdot 1 \%$ ), skin/soft-tissue abscesses, arthritis or osteomyelitis (44 patients, $28 \cdot 1 \%$ ), empyema or lung abscesses (32 patients including one with both empyema and intra-abdominal infection, 20\%) and intracranial abscesses (15 patients, 9.4\%). Most patients with non-pyogenic infections had bacteraemia (70, $68 \%$ ); none had endocarditis. Patients with PIs were generally younger and had fewer co-morbidities (Table 1). Most infections were acquired in the community, similarly in both groups. Surgical procedures were performed for abscess or empyema drainage in most patients with PI $(141 / 160,88 \cdot 1 \%)$. Infection severity and mortality were higher in patients with non-pyogenic infections.

PIs were observed most commonly with $S$. intermedius $(31 / 36,86 \cdot 1 \%)$ compared with $57 \cdot 1 \%(104 / 182)$ with $S$. anginosus and $55 \cdot 6 \%(25 / 45)$ with $S$. contellatus, $P=0 \cdot 04$. The final multivariate model predicting PIs included age, baseline cognitive or functional impairment, acute mental alternation and raised BUN at infection presentation and SAG species. Congestive heart failure, diabetes and other laboratory values failed to improve the model. S. anginosus (OR 0.18, 
Table 1. Patient characteristics and outcomes of patients with pyogenic vs. non-pyogenic SAG infection

\begin{tabular}{|c|c|c|c|}
\hline & $\begin{array}{l}\text { Pyogenic infection } \\
N=160\end{array}$ & $\begin{array}{l}\text { Non-pyogenic infection } \\
N=103\end{array}$ & $P$ value \\
\hline \multicolumn{4}{|l|}{ Demographics and background conditions } \\
\hline Mean age, years (s.D.) & $52 \cdot 3(18 \cdot 8)$ & $63 \cdot 8(17 \cdot 4)$ & $>0 \cdot 001$ \\
\hline Male $(\%)$ & $104(65 \%)$ & $64(62 \cdot 1 \%)$ & $0 \cdot 366$ \\
\hline Heart failure (\%) & $13(8 \cdot 1 \%)$ & $16(15 \cdot 7 \%)$ & $0 \cdot 057$ \\
\hline Diabetes mellitus (\%) & $23(14 \cdot 4 \%)$ & $31(30 \cdot 1 \%)$ & 0.02 \\
\hline COPD $(\%)$ & $4(2 \cdot 5 \%)$ & $4(3 \cdot 9 \%)$ & 0.52 \\
\hline Connective tissue disease (\%) & $7(4 \cdot 4 \%)$ & $2(1 \cdot 9 \%)$ & $0 \cdot 29$ \\
\hline Dementia $(\%)$ & $6(3 \cdot 8 \%)$ & $15(14 \cdot 6 \%)$ & 0.02 \\
\hline Liver disease $(\%)$ & $13(8 \cdot 1 \%)$ & $8(7 \cdot 8 \%)$ & $0 \cdot 9$ \\
\hline Cognitive/physical impairment (\%) & $16(10 \%)$ & $24(23 \cdot 3 \%)$ & 0.03 \\
\hline Active malignancy $(\%)$ & $34(21 \cdot 2 \%)$ & $26(25 \cdot 2 \%)$ & $0 \cdot 45$ \\
\hline Community-acquired infection (\%) & $129(80 \cdot 6 \%)$ & $82(78 \cdot 6 \%)$ & $0 \cdot 85$ \\
\hline \multicolumn{4}{|l|}{ Infection presentation and characteristics } \\
\hline SAG species & & & $0 \cdot 004$ \\
\hline Streptococcus anginosus & $104(65 \%)$ & $78(75 \cdot 7 \%)$ & \\
\hline Streptococcus constellatus & $25(15 \cdot 6 \%)$ & $20(19 \cdot 4 \%)$ & \\
\hline Streptococcus intermedius & $31(19 \cdot 4 \%)$ & $5(4 \cdot 9 \%)$ & \\
\hline Polymicrobial infection (\%) & $102(63 \cdot 8 \%)$ & $66(64 \cdot 1 \%)$ & $0 \cdot 95$ \\
\hline Bacteraemia & $13(8 \cdot 1 \%)$ & $70(68 \%)$ & $<0 \cdot 001$ \\
\hline Acute renal failure $(\%)$ & $11(6 \cdot 9 \%)$ & $11(10 \cdot 7 \%)$ & $0 \cdot 27$ \\
\hline Tachycardia (\%) & $63(39 \cdot 4 \%)$ & $42(41 \cdot 2 \%)$ & $0 \cdot 77$ \\
\hline Hypotension (\%) & $28(17 \cdot 5 \%)$ & $36(35 \cdot 3 \%)$ & $0 \cdot 01$ \\
\hline Mental alternation (\%) & $11(6 \cdot 9 \%)$ & $17(16 \cdot 5 \%)$ & $0 \cdot 01$ \\
\hline WBC median (IQR 25-75) & $13(9 \cdot 5-17)$ & $12(9-16)$ & $0 \cdot 23$ \\
\hline Platelet, median (IQR 25-75) & $255 \cdot 5(198-381)$ & $248 \cdot 5(173-359)$ & $0 \cdot 34$ \\
\hline Blood urea nitrogen $(\mathrm{mg} / \mathrm{dl})$ median (IQR 25-75) & $13(9-19 \cdot 5)$ & $19(14-32)$ & $0 \cdot 01$ \\
\hline Creatinine, median (IQR 25-75) & $0 \cdot 84(0 \cdot 7-1 \cdot 1)$ & $1(0 \cdot 73-1 \cdot 43)$ & $0 \cdot 11$ \\
\hline \multicolumn{4}{|l|}{ Clinical outcomes } \\
\hline Lengths of hospitalisation (days), median (IQR 25-75) & $10(6-20)$ & $12(6-20)$ & $0 \cdot 7$ \\
\hline In hospital mortality $(\%)$ & $14(8 \cdot 8 \%)$ & $25(24 \cdot 3 \%)$ & $0 \cdot 01$ \\
\hline 30 days mortality $(\%)$ & $15(9 \cdot 4 \%)$ & $30(29 \cdot 1 \%)$ & $>0 \cdot 001$ \\
\hline
\end{tabular}

$95 \%$ CI $0 \cdot 06-0 \cdot 53$ ) and S. constellatus (OR 0.14, 95\% CI 0.04-0.48) were associated with significantly fewer PIs compared with $S$. intermedius; age was the only other significant factor associated with PIs (Table 2). The model performance was adequate; HosmerLemeshow $P=0 \cdot 82$, area under the ROC curve 0.74 $(95 \%$ CI $0 \cdot 67-0 \cdot 8)$.

Over the course of 7 years, we identified 263 patients with clinically significant SAG infections with $S$. anginosus being the most frequent species (69\%) isolated. Overall, there was a relatively high proportion of PIs $(60 \%)$ and these were associated with younger and healthier patients without bacteraemia, while bacteraemia occurred more frequently in patients without a PI. S. intermedius caused significantly more PIs than other SAG species, when adjusted to other differences between patients with and without pyogenic infections.
Younger age remained significantly associated with PIs in the adjusted analysis. Survival was higher for patients with PIs, relating to the lower rate of bacteraemia in this group, younger age, fewer co-morbidities and possibly because drainage was performed in most of patients.

In agreement with our study, there is an evolving understanding that $S$. intermedius is more invasive and causes more severe infections than the other SAG species. In a literature review of 12 patients with SAG-disseminated infections (defined as involving two or more major organs), $S$. intermedius was responsible for six infections, while $S$. anginosus and S. constellatus caused two each [6]. Likewise, a retrospective single-centre study including 118 patients with SAG infections, $S$. intermedius was the least common but resulted in the highest rate of abscess 
Table 2. Risk factors for pyogenic infection, multivariate analysis

\begin{tabular}{lll}
\hline \hline $\begin{array}{l}\text { Factors predicting } \\
\text { pyogenic infection }\end{array}$ & OR $(95 \% \mathrm{CI})$ & $P$ value \\
\hline SAG species & & \\
$\quad$ Streptococcus intermedius & Reference & \\
$\quad$ Streptococcus anginosus & $0 \cdot 18(0 \cdot 06-0 \cdot 53)$ & 0.02 \\
$\quad$ Streptococcus constellatus & $0.14(0 \cdot 04-0 \cdot 48)$ & 0.02 \\
Acute mental alteration & \\
Blood urea nitrogen $(\mathrm{mg} / \mathrm{dl})^{\mathrm{a}}$ & $0.41(0 \cdot 16-1 \cdot 04)$ & 0.06 \\
Age (years) $^{\text {Age }}$ & $0.97(0.96-1)$ & 0.08 \\
\hline \hline
\end{tabular}

${ }^{\text {a }}$ Measured at onset of infection.

formation (10/12, $88 \%$ of patients), compared with 41/ $54(76 \%)$ with $S$. constellatus and 10/52 (19\%) with $S$. anginosus, and a higher rate of bacteraemia [4]. In our study, S. intermedius was similarly associated with a higher rate of PIs (using a broader definition than abscesses), but was associated with highly significantly lower rates of bacteraemia than $S$. constellatus and $S$. anginosus.

The reasons for the increased pyogenic potential of $S$. intermedius have not been fully elucidated. Genetic mutations resulting in overproduction of intermedilysin, a major virulence factor of SAG, has been described [9], while the presence and activity of hydrolytic enzymes (deoxyribonuclease and chondroitin sulfatase) have been reported to be present more frequently $S$. intermedius and $S$. constellatus, and were associated with infection-related strains [10].

Our study, as most of the reviewed literature, is a retrospective single-centre study with its inherent limitations. The high proportion of PIs reported here may be explained by the fact that we included only clinically significant infections, unlike other studies with lower rates $(35-51 \%)$ of such infections [3, 4]. We also employed a broader definition for 'pyogenic infections' and the long time span of the study enabled the collection of a relatively large series of patients, therefore allowing us to adjust for confounders when assessing the relative contribution of the individual SAG species to PIs. Throughout this period, the Vitek 2 system was used to identify the SAG isolates to the species level. Although currently molecular identification might be considered more accurate, several studies have confirmed the good performance of Vitek-2 in SAG identification [3, 11-13].

In conclusion, our findings suggest that compared with other SAG species, S. intermedius has the highest potential to cause infections involving abscess formation or other deep-seated infections and its identification should trigger an investigation to identify foci requiring surgical interventions. S. constellatus and $S$. anginosus caused bacteraemia without an associated pyogenic infection more frequently than $S$. intermedius.

\section{ACKNOWLEDGEMENTS}

The authors would like to acknowledge the contribution of the ESCMID Study Group for Infections in the Elderly (ESGIE) for the inspiration and motivation to conduct the study. The study was performed without funding.

\section{DECLARATION OF INTEREST}

None.

\section{REFERENCES}

1. Laupland KB, et al. Population-based surveillance of invasive pyogenic streptococcal infection in a large Canadian region. Clinical Microbiology and Infection 2006; 12: 224-230.

2. Gossling J. Occurrence and pathogenicity of the Streptococcus milleri group. Reviews of infectious Diseases 1988; 10: 257-285.

3. Siegman-Igra Y, Azmon Y, Schwartz D. Milleri group streptococcus - a stepchild in the viridans family. European Journal of Clinical Microbiology \& Infectious Diseases 2012; 31: 2453-2459.

4. Claridge JE, et al. Streptococcus intermedius, Streptococcus constellatus, and Streptococcus anginosus ('Streptococcus milleri group') are of different clinical importance and are not equally associated with abscess. Clinical Infectious Diseases 2001; 32: 1511-1515.

5. Junckerstorff RK, Robinson JO, Murray RJ. Invasive Streptococcus anginosus group infection-does the species predict the outcome? International Journal of Infectious Diseases 2014; 18: 38-40.

6. Simone G, et al. Streptococcus anginosus group disseminated infection: case report and literature review. Le Infezioni in Medicina 2012; 3: 145-154.

7. Bone RC, et al. American College of Chest Physicians/ Society of Critical Care Medicine Consensus Conference: definitions for sepsis and organ failure and guidelines for the use of innovative therapies in sepsis. Critical Care Medicine 1992; 20: 864-874.

8. Horan TC, Andrus M, Dudeck MA. CDC/NHSN surveillance definition of health care-associated infection and criteria for specific types of infections in the acute care setting. American Journal of Infection Control 2008; 36: 309-332. doi: 10.1016/j.ajic.2008.03.002. 
9. Tomoyasu T, et al. Lacr mutations are frequently observed in Streptococcus intermedius and are responsible for increased intermedilysin production and virulence. Infection and Immunity 2013; 81: 3276-3286.

10. Jacobs JA, Stobberingh EE. Hydrolytic enzymes of Streptococcus anginosus, Streptococcus constellatus and Streptococcus intermedius in relation to infection. European Journal of Clinical Microbiology \& Infectious Diseases 1995; 14: 818-820.

11. Chatzigeorgiou KS, et al. Phoenix 100 versus Vitek 2 in the identification of Gram-positive and Gram-negative bacteria: a comprehensive meta-analysis. Journal of Clinical Microbiology 2011; 49: 3284-3291.

12. Haanpera $\mathbf{M}$, et al. Identification of alpha-hemolytic streptococci by pyrosequencing the 16S rRNA gene and by use of VITEK 2. Journal of Clinical Microbiology 2007; 45: 762-770.

13. Funke G, Funke-Kissling P. Performance of the new VITEK 2 GP card for identification of medically relevant Gram-positive cocci in a routine clinical laboratory. Journal of Clinical Microbiology 2005; 43: 84-88. 